\title{
AN INVESTIGATION OF THE ABSORPTION OF ULTRAVIOLET LIGHT BY GEREBROSPINAL FLUID IN VARIOUS DISEASE STATES
}

\author{
BY
}

\author{
E. FRETSON SKINNER, ShefField
}

\section{INTRODUCTION}

THE examination of cerebrospinal fluid in various diseased states has become a matter of routine investigation and at times yields information of great value in particular cases-for instance, the presence of a cellular content of over 3 per c.mm. leads to a suspicion of some infective process involving the central nervous system, and the preponderance of a mononuclear type of cell suggests the probability of such infection being of a granulomatous nature, syphilis or tuberculosis. Further, an increase in the protein content of the fluid is indicative of chronic infection, while reduction in the amount of sodium chloride present below 0.6 per cent. is almost pathognomonic of tuberculous meningitis.

Of the more recent tests used in the examination of cerebrospinal fluid the precipitation of gold from a colloidal suspension of gold chloride is of peculiar interest. It appears to be an extremely sensitive test and to possess a definite diagnostic value. It is not necessary to refer in detail to this test; suffice it to say that it appears to depend on the upset of the balance of electric charges on the colloid particles in the gold solution with consequent precipitation of metallic gold. Lange's work at first seemed to suggest that in this method was to be found a specific test for neurosyphilis, since the highly albuminous fluids of general paralysis and tabes caused precipitation of gold in a manner which could be expressed as a simple curve. Other workers confirmed Lange's observations, but as the test became more widely recognized it was soon found that cerebrospinal fluids from cases other than neurosyphilis caused a similar precipitation, notably disseminated sclerosis. The test lost its specificity as a touchstone for neurosyphilis, but none the less the curves obtained are so characteristic that in association with other tests it is possible to say with considerable certainty whether a particular type of curve is caused by a fluid from a case of neurosyphilis or of disseminated sclerosis.

\section{PERSONAL INVESTIGATIONS}

It was the consideration of this type of reaction, depending as it does on some physical characteristic of the fluid-either the state of the colloid 
contents or the electrolytes-that led me to speculate on the chances of finding some physical method of examination of cerebrospinal fluid whereby its varying constitution might be evidenced, and, after trying one or two methods, the effect of absorption of ultraviolet waves seemed to offer the best likelihood of success.

Early in 1927, therefore, I began a series of observations on the absorption of the ultraviolet waves, using a very primitive layout; the results, though purely qualitative, appeared to me to be sufficiently definite to warrant a more serious investigation. In consequence, I purchased from Messrs. Adam Hilger one of their new photometers, and during the last two or three years as time and opportunity have offered I have continued to make observations on the cerebrospinal fluid, the results of which are set out below.

The earlier observations made with a small spectrograph and a homemade optical bench, arc, and quartz-cell holder, etc., were put together in two short papers which appeared in this Journdl in October, 1929, and December, 1930.

At this time, so far as I could ascertain, there was no literature on the subject, though from a private source I heard that Campbell Smith had been observing the absorption of ultraviolet waves. I went to see him and he very kindly showed me some of his curves which dealt with spectroscopy of filtered blood serum.

Since that time one or two continental observers have made observations on these lines and their results will be referred to later in this paper.

In my early observations I had no means of calibrating my results, which were only expressible in terms of the wavelength at which absorption began; but the appearance of the absorption in cases of tuberculous meningitis was so striking that I felt justified in concluding that tuberculous fluids might be diagnosed from these spectroscopic tests. They were almost constant, but later I found one or two cases in which absorption was not so complete, so I was compelled to modify my early view, and consider that only in the later stages of the disease did such complete absorption occur. Even in the first few days of the disease, however, absorption commences earlier in the scale than in any other condition I have examined.

The only other fluid which appeared sufficiently characteristic to be easily recognized was that from cases of general paralysis of the insane, and here again the amount of absorption seemed to depend on the length of time the disease had been present.

While it was clear that such changes could not be diagnostic of any definite clinical picture and that they represented varying states of the cerebrospinal fluid, some of them might be sufficiently constant as to be suggestive of clinicopathological events. For instance, I felt that early in tuberculous meningitis absorption might be found somewhere about $\lambda 2397$; but with a further area of absorption between $\lambda 3051$ and $\lambda 2764$, later in the 
course of the case, the lines seen in the spectrogram between $\lambda 2764$ and $\lambda 2397$ tended to disappear, while in other forms of meningitis in which recovery took place the absorbed lines between $\lambda 3051$ and $\lambda 2764$ tended to reappear.

This was one of the conclusions at which $I$ arrived in 1928, namely that in meningitic conditions the cerebrospinal fluid gave a varying spectrogram, but one which could be recognized and perhaps serve as some help in prognosis. 'It is, of course, perfectly clear that such changes as I am describing in one tissue, cerebrospinal fluid, cannot carry a diagnostic label; our labels are too gross for such minuteness. For instance, the label " general paralysis" conveys to our minds a more or less definite clinical picture, but the picture is really a moving one inasmuch as we envisage a series of events occurring in time, whereas an examination such as we are making can only show the results of certain metabolic accidents at a given moment, and such results as I have obtained must vary at different stages within the range of one disease-process. It is manifestly absurd to think, for instance, that every case of general paralysis must give the so-called paretic gold curve in Lange's test since the factors on which this test depend are probably only progressively developed. The results I have obtained spectrographically, if they have any value at all, can only suggest that certain tendencies are at work and can never have the same evidential value for a given instant as, say, the X-ray picture of a fractured bone.' 1

It appeared to me from these early observations that such a method of investigating cerebrospinal fluid was worth more extended trials, for I could not but feel that there must be some difference in constitutions of cerebrospinal fluid in varying bodily conditions ' when one looks at the perfectly clear fluid from a case of tuberculous meningitis and an equally clear fluid from a case of tabes or general paralysis and in both finds a pleocytosis, an increased protein content, an identical chloride content, yet with very different spectrograms. The conclusion seems legitimate either that our means of chemical investigation are too gross to detect the minute differences in composition on which the spectrograms are dependent or, what is more likely, these differences depend on factors other than chemical., ${ }^{2}$

\section{METHODS}

The principle of ultraviolet spectrophotometry consists of photographing two spectra one immediately below the other so that any absorbed line in the one spectrum may be compared with a similar line in the other. One of these spectra is that produced by light which is passed through the solution to be analysed, the other through a solvent such as distilled water. The source of light, e.g. the spark from an induced current sparking between electrodes of tungsten, copper, iron or other metal, gives one of the wellknown ' line spectra,' and such spectra are photographically recorded and 
the absorption of any of these lines is shown by its absence from the spectrum.

In spectrographic observations great use is made of a factor known as the 'extinction coefficient,' the value of which has been arrived at as follows. According to Lambert's law ' the proportion of light absorbed by a substance is independent of the intensity of the incident light,' just as a sponge can only absorb a certain amount of water irrespective of the volume or the velocity with which the water impinges on the sponge.

If $J_{0}$ be the intensity of light entering a medium and $J$ the intensity after passing through a length of $d$ centimetres, and if $1 / a$ be that length of path, the passage through which reduces the light intensity to $1 / 10$ th, then

$$
\mathrm{J}=\mathrm{J}_{\mathrm{o}} \cdot 10^{-a d} \text { or } \frac{\mathrm{J}_{\mathrm{o}}}{\mathrm{J}}=10^{a d}
$$

where $a-a$ constant depending on the medium-is called the extinction coefficient.

This is the definition of the extinction coefficient given by Bunsen and Roscoe, and from the formula it will be seen that it is the reciprocal of the thickness of the medium which is necessary to reduce the light to $1 / 10$ of its incident value

$$
\left(a=\frac{1}{d} \log \frac{\mathbf{J}_{0}}{\mathbf{J}}\right)
$$

A further term is useful, namely the 'density.' If $J$ and $J_{0}$ have the significance given above, we may write $J=J_{0} 1^{-D}$, D being called the 'density.'

$$
\mathbf{J}=\mathbf{J}_{\mathbf{0}} 10^{-\mathrm{D}}, \quad \mathbf{J}=\frac{\mathbf{J}_{0}}{10^{\mathbf{D}}}, \quad \mathbf{D}=\log \frac{\mathbf{J}_{\mathrm{o}}}{\mathbf{J}} .
$$

Therefore $\mathbf{D}=a d$; that is, the density is the product of the extinction coefficient and the depth of the medium (in centimetres).

It is necessary to define these terms, since unfortunately they are used occasionally with other meanings, the meanings here assigned being those given by Bunsen and Roscoe" to the extinction coefficient and by Hurter and Driffield ${ }^{4}$ to density.

The method of recording the absorption in these experiments is the one usually employed in this country, and consists in determining the points in the spectrum at which absorption occurs and expressing on a graph these points in terms of density and wavelength, using the former as ordinates and the latter as abscissæ.

The practical application of these theoretical considerations has proved of great use in identifying substances in solution, and although no clinical use has been made of them so far, I consider the method may eventually be found to have some importance, particularly in medicolegal work, where the spectrophotometric examination of body fluids might disclose the presence of soluble poisons. 


\section{TECHNIQUE}

The actual observations are not difficult once the apparatus has been set up, though admittedly the apparatus is amongst the most delicate in use and requires some skill in adjusting, but once adjusted, observations can be mate a simple routinc procedure. Roughly speaking, the technique is as follows. Light is passed through the medium under examination as well as through an indifferent medium, and the resulting beams, which are parallel, are analysed by quartz prisms. The resulting spectra are received on photographic plates. Various methods have been adopted for controlling the results, but whatever method is employed the result is the same-two spectral bands, one of which is produced by light passing through the medium to be analysed, the other through the indifferent medium, are received on a

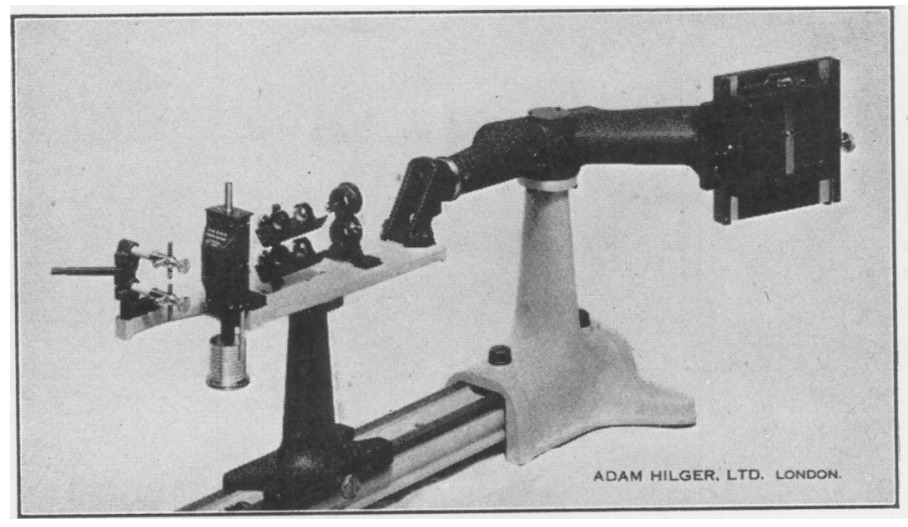

Fig. 1.*

photographic plate. The amount of light through the first is fixed, while that through the latter is variable.

When the resulting photographs of the spectral bands are examined it is clear that the photographic blackening of each line depends on the amount of light reaching the plate at that point, and if the light passing through the medium is absorbed this photographic blackening will be diminished. In two spectra juxtaposed, if it be found that two lines are of equal blackness it is proof that similar amounts of light have passed through the medium under examination and the control medium.

If any solution of electrolytes is used for analysis and distilled water for the control, the resulting spectra will show a certain amount of 'selective' absorption, i.e. lines will be diminished in blackness in the spectrum of the light passing through the solution of the electrolytes. The corresponding lines in the spectrum of the light passing through the distilled water will be

* This is a later model than the one used in the experiments. 
much darker. The various forms of apparatus are designed so that this latter beam may be so reduced in intensity that the diminished blackening produced by the absorption may be matched by reduction of light passing through the distilled water. As has been said bcfore, when any two lines are of equal blackness it is because the amount of light falling on the photographic plate is the same in both cases ; in the one case reduction is caused by absorption, in the other by reduction of light intensity. One can therefore measure the absorption by the diminution in light intensity, and thus absorption can be expressed in terms of intensity of light so measured.

The photometric method I have used in my observations is one in which this relationship is of the simplest.

The apparatus, the Spekker photometer, is seen in fig. 1.

It consists of a pair of electrodes for the spark, the light from which is passed through reflecting prisms whereby the single beam is split into two,

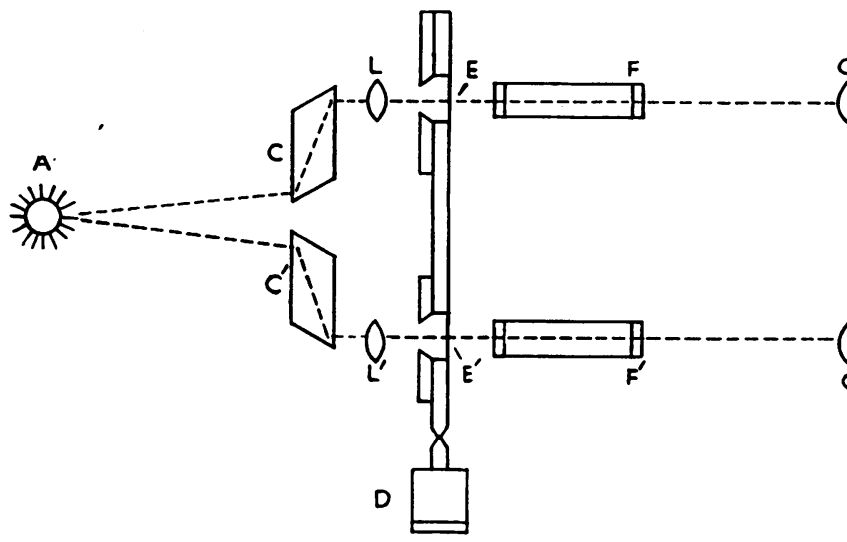

Fig. 1A.

one of which passes through the medium under examination, the other through the control medium, after which the two beams are again brought close together and directed, still parallel, into the spectrograph.

Light emanating from source $\mathbf{A}$ enters the quartz reflecting prism $\mathbf{C}$ and after undergoing a further reflection is rendered parallel by the lens $\mathbf{L}$. This parallel beam of light, after traversing the medium $F$ of which the absorbent constants are required, falls on the lens $G$, which focusses the light, after being reflected by the prism $\mathbf{H}$, on to the slit of the spectrograph, forming there an image of one-half of the light source. A similar cone of light falls on the prism $C^{\prime}$, is rendered parallel by $L^{\prime}$, and after passing through the control medium $F^{\prime}$ is focussed by the lens $G^{\prime}$, forming an image of the other half of the source of light on the slit of the spectrograph after reflection by the prism $\mathbf{H}^{\prime}$.

Immediately in front of the lenses $L$ and $L^{\prime}$ are placed two rectangular apertures $\mathbf{E}$ and $\mathbf{E}^{\prime}$, controlling the amount of light passing through the 
photometer. The aperture $\mathbf{E}$ in the upper path is fixed in area while the lower aperture $\mathbf{E}^{\prime}$ can be varied at will to give any area of opening. This adjustment is achieved by turning the drum $\mathrm{D}$, which by means of an accurate screw alters the position of one of the sides of this variable aperture.

Thus the spectrograph is illuminated by two beams of light of the same origin, the intensity of one being varied by the absorbing medium under test, while the intensity of the other beam is controlled by the opening of the variable aperture. In any photograph of a spectrum (spectrogram) taken under the above conditions, the points at which the lines are of equal blackening indicate that the light passing through the material under test is equal to that which has passed through the variable aperture.

When no absorbing medium is present the spectra are of equal intensity only when the areas of the fixed and variable apertures are the same, the ratios of the respective areas being a measure of the ratios of the intensities of the two beams of light. It can easily be shown that the intensities of the two beams are proportional to the respective areas of the fixed and variable apertures.

Let $J_{0}$ be the intensity of the light entering the absorbing medium and $\mathbf{J}$ the intensity after transmission through the medium. Then since the areas of the apertures are proportional to the light transmitted and since also at the points where there is equal blackening of the lines the area of the variable aperture has been adjusted to make the intensity of the control beam equal to that of the light after passing through the medium, the ratio $\frac{\mathbf{J}_{0}}{J}$ may be represented by the expression

$$
\frac{\mathbf{J}_{\mathbf{o}}}{\mathbf{J}}=\frac{\text { area of fixed aperture }}{\text { area of variable aperture }} .
$$

The drum $\mathbf{D}$ which controls the variable aperture has on it an engraved scale to read $\log \frac{\mathbf{J}_{0}}{\mathbf{J}}$, which logarithm, as has been mentioned above, is referred to as the density.

By taking a series of photographs with the drum set to different density values and then on the negative searching for the points of equal blackening of the lines in the two spectra, one is able to obtain a number of values of $\frac{\mathbf{J}_{0}}{\mathbf{J}}$ for a wide range of wavelengths and from the values so obtained to construct a graph connecting the density $\left(\log \frac{\mathbf{J}_{0}}{J}\right)$ with the wavelength.

It will be seen from the above considerations that in order to obtain an absorption curve of cerebrospinal fluid all that is necessary, using the Spekker photometer, is to take a series of bipartite spectra on one plate with various density readings and then plot out the points at which the lines are of equal blackness. 
The rest of my apparatus consisted of an old 32-inch X-ray induction coil with a plate condenser of 0.005 microfarad capacity in parallel with the spark, the electrodes being of tungsten steel. The whole was energized by a direct current of 60 volts interrupted by a turbine motor break working direct from the A.C. mains (250 volts). The spectrograph was fitted directly to the photometer.

The exposure depends on the source of light. The best results are obtained by making it of constant actinic value by increasing the time according to the expression $\mathbf{E}=\mathbf{K}$ antilog $\mathbf{D}$, where $\mathrm{K}$ is a constant depending on the source of light and $D$ is the density.

By trial and error $I$ found 3 seconds to be the best value for $K$, so that my exposure table for a single observation was as follows :-

\begin{tabular}{c|c|c|c}
\hline Density & Antilog & Exposure calculated & Exposure used \\
\cline { 2 - 3 } $0 \cdot 0$ & $1 \cdot 0$ & $3 \cdot 0$ & 3 \\
$0 \cdot 1$ & $1 \cdot 26$ & $3 \cdot 78$ & 4 \\
$0 \cdot 2$ & $1 \cdot 58$ & $4 \cdot 74$ & 5 \\
$0 \cdot 3$ & $2 \cdot 0$ & $6 \cdot 0$ & 6 \\
$0 \cdot 4$ & $2 \cdot 51$ & $7 \cdot 53$ & 8 \\
$0 \cdot 5$ & $3 \cdot 16$ & $9 \cdot 48$ & 10 \\
$0 \cdot 6$ & $3 \cdot 98$ & $11 \cdot 94$ & 12 \\
$0 \cdot 7$ & $5 \cdot 01$ & $15 \cdot 03$ & 15 \\
$0 \cdot 8$ & $6 \cdot 31$ & $18 \cdot 9$ & 20 \\
$0 \cdot 9$ & $7 \cdot 98$ & $23 \cdot 94$ & 25 \\
$1 \cdot 0$ & $10 \cdot 0$ & $30 \cdot 0$ & 30 \\
$1 \cdot 1$ & $12 \cdot 59$ & $37 \cdot 77$ & 35 \\
$1 \cdot 2$ & $15 \cdot 85$ & $47 \cdot 65$ & 45 \\
$1 \cdot 3$ & $19 \cdot 95$ & $59 \cdot 85$ & 55 \\
$1 \cdot 4$ & $25 \cdot 1$ & $75 \cdot 3$ & 75 \\
$1 \cdot 5$ & $31 \cdot 6$ & $94 \cdot 8$ & 95 \\
$1 \cdot 6$ & $39 \cdot 8$ & $119 \cdot 4$ & 120 \\
$1 \cdot 7$ & $50 \cdot 1$ & $150 \cdot 3$ & 150 \\
$1 \cdot 8$ & $63 \cdot 1$ & $189 \cdot 3$ & 190 \\
$1 \cdot 9$ & $79 \cdot 8$ & $239 \cdot 4$ & 240 \\
$2 \cdot 0$ & $100 \cdot 0$ & $300 \cdot 0$ & 300 \\
& & & \\
\hline
\end{tabular}

Ilford special rapid plates were used throughout and were developed by time and temperature method.

After a negative is finished, the next step is to 'spot' the points where the two lines in the adjoining spectra are of the same blackness. This is done by placing the negative on a viewing-box suitably illuminated with a face of opal glass, and with a fine pen marking the points on the negative with Indian ink.

When all the spectra on one negative have been so 'spotted' (usually 15 or 16 exposures on one plate) the positions of these ink dots can be read off on a wavelength scale. From each negative at the end of this process 
of spotting a table of figures is obtained composed of the wavelengths of the absorbed lines at a particular density, and with these two values a graph is made in each case.

\section{ABSORPTION GURVE OF NORMAL GEREBROSPINAL FLUID}

It was first essential to examine a specimen of normal cerebrospinal fluid, and having acquired a constant technique I made a number of observa-

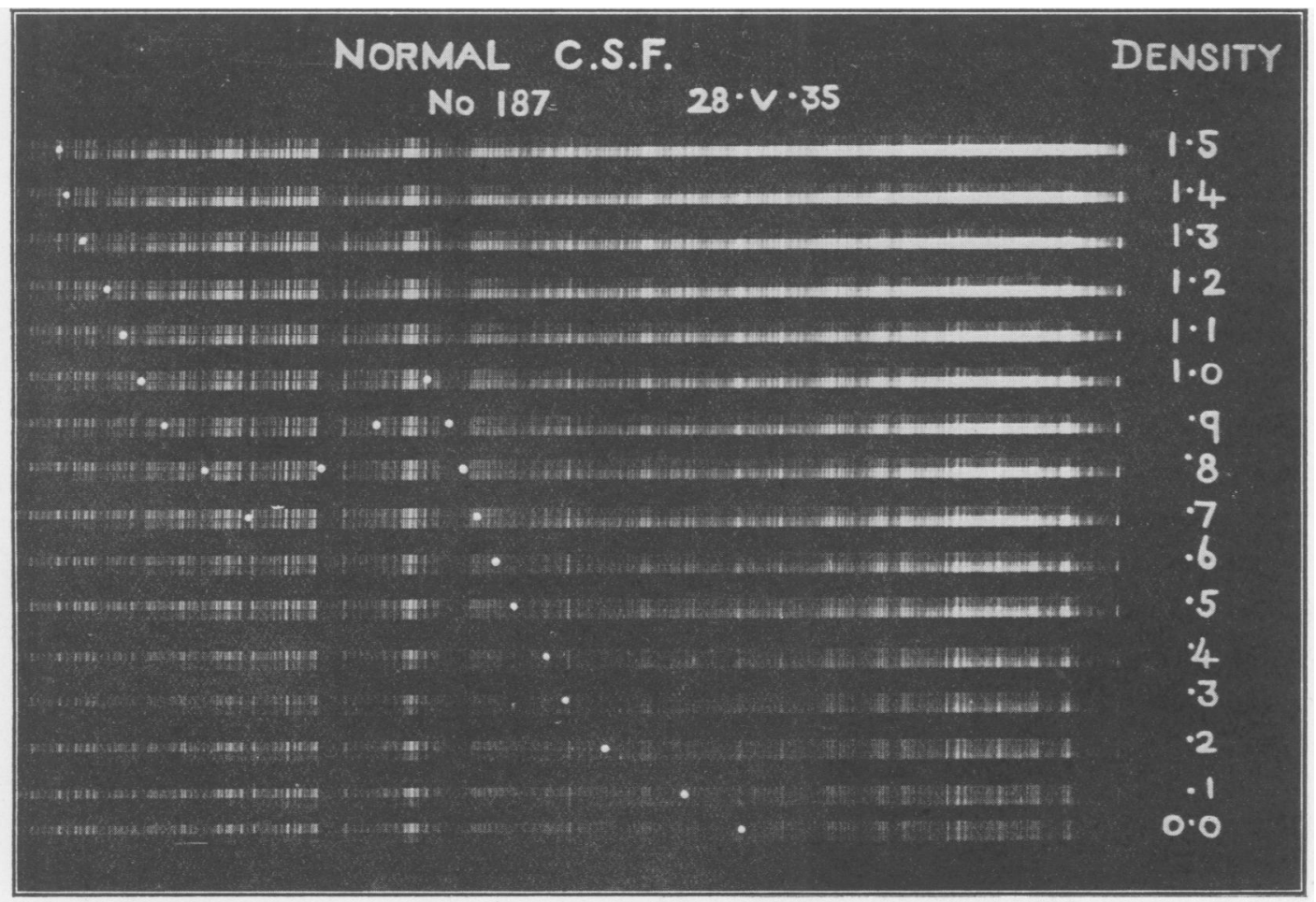

Fig. 2.

tions on normal fluids obtained from volunteers from the local Unemployment Exchange. Fig. 2 is the print of an absorption spectrogram given by normal fluid.

The 'spots' are placed on contiguous lines of similar photographic density and for the purpose of reproduction have been made unnecessarily big. Four such negatives have been used in preparing the composite graph shown in fig. 3, and it will be seen that all show absorption of almost identical kind, the variations in the graph being relatively slight.

From a study of such negatives and graphs it is clear that there is complete absorption of all waves shorter than those of $\lambda 2380$, while waves 
of a length of $\lambda 2630$ are only slightly absorbed; more absorption is seen in the neighbourhood of wavelength $\lambda 2650$ and $\lambda 2800$ and so on. Thus, examination of a spectrogram of normal cerebrospinal fluid is found to give evidence of some selective absorption between wavelength $\lambda 2380$ and $\lambda 3200$.

This selective absorption is demonstrated in the graph in fig. 3 , where it will be noticed that the curve is broken by a ' hump' in the middle of its course. The position of this hump with its subsequent depression and following rise is the characteristic feature of the 'absorption curve' of

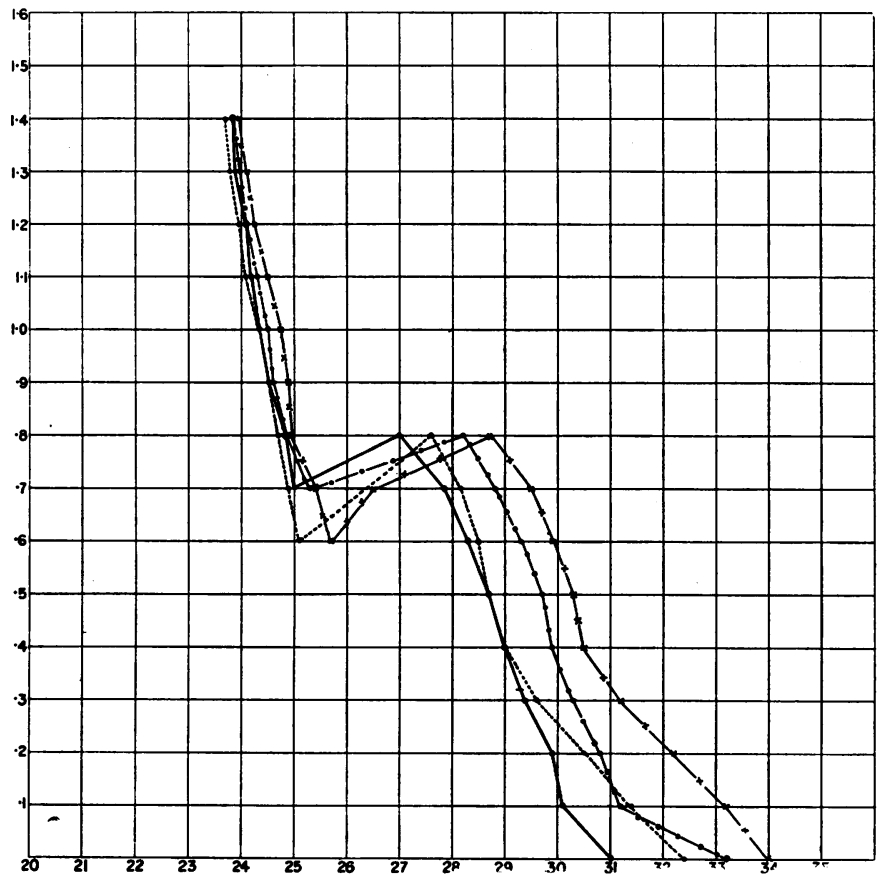

Fig. 3.-Normal cerebrospinal fluids. Composite graph from four negatives showing normal limits of variation in absorption of ultraviolet waves. Fluid obtained from four healthy persons.

cerebrospinal fluid, and, as will be seen presently, the striking differences between normal fluids and those from cases suffering from a variety of different diseased states are generally found in a particular region of the scale between $\lambda 3250$ and $\lambda 2380$.

\section{PATHOLOGIGAL ABSORPTION GURVES}

Having determined what appeared to be the normal absorption curve of cerebrospinal fluid, I proceeded to investigate specimens from cases in which it was being examined in the laboratory in the course of routine clinical work. My first 50 or 60 cases were unselected. I received from various sources all kinds of cerebrospinal fluids, from cases of suspected cerebral tumour, myelitis, meningitis, chorea, syphilis of the central nervous system, 
etc., and I would here like to thank those of my colleagues, both at my own hospital and others in the city, who were kind enough to send me material for my investigation.

A study of the absorption of ultraviolet waves in this first series of 50 cases made it clear that one or two tentative conclusions could be drawn.

1. Cases of tuberculous meningitis gave rise to a characteristic change in the curve, as did also

2. Cases of general paralysis of the insane, but here the change was not so striking.

3. During the course of a disease the curve tended to become steeper if progress was towards fatality.

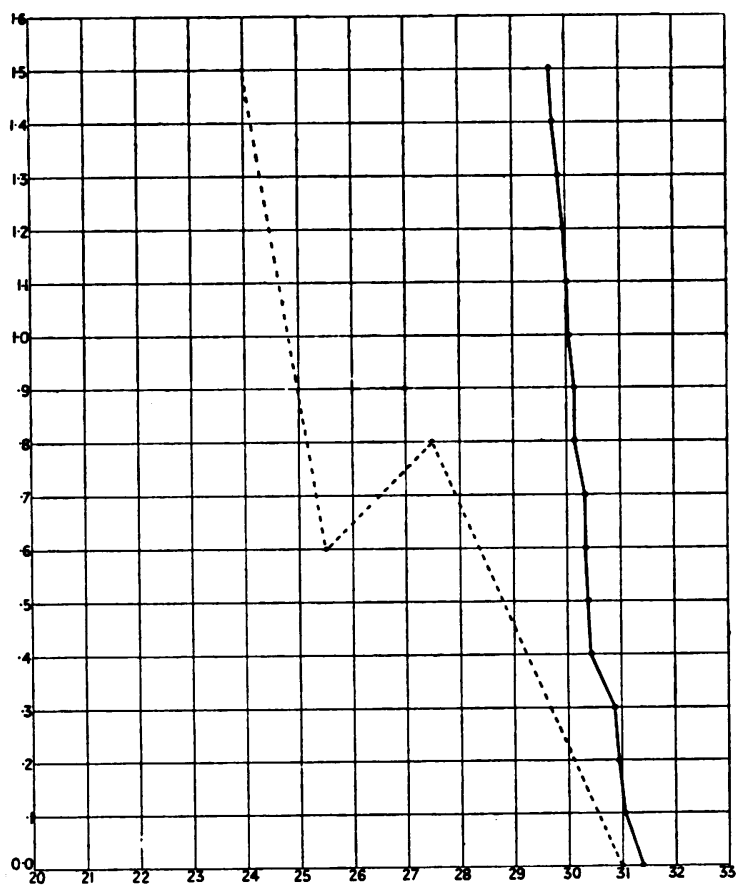

Fig. 4.-Tuberculous meningitis, late stage.

These observations tended to confirm my previous conclusions in the two papers mentioned above.

When the terms tuberculous meningitis or general paralysis are used, it must of course be understood that such can only be rough clinical labels, and an examination such as the one under discussion can only refer to a particular biochemical state at a particular time, since during the plogress of a diseased condition such biochenicel states must vary. It is surprising to find however that the biochemistry of the cerebrospinal fluid does remain remarkably constant. 
Fig. 4 is a print from a case of (clinical) tuberculous meningitis verified at autopsy. It will be noted that there is a striking total absorption producing an almost vertical graph (within the limits of my observational technique), though there is at times in similar cases a suggestion of a hump much higher in the graph, as is also seen in cases of general paralysis at times.

In fig. 5 a few lines appear between $\lambda 2650$ and $\lambda 2500$, but only when the coefficient of extinction is high (1.3), and I have little doubt that even this part of the spectrogram would be completely absorbed in a few days (in

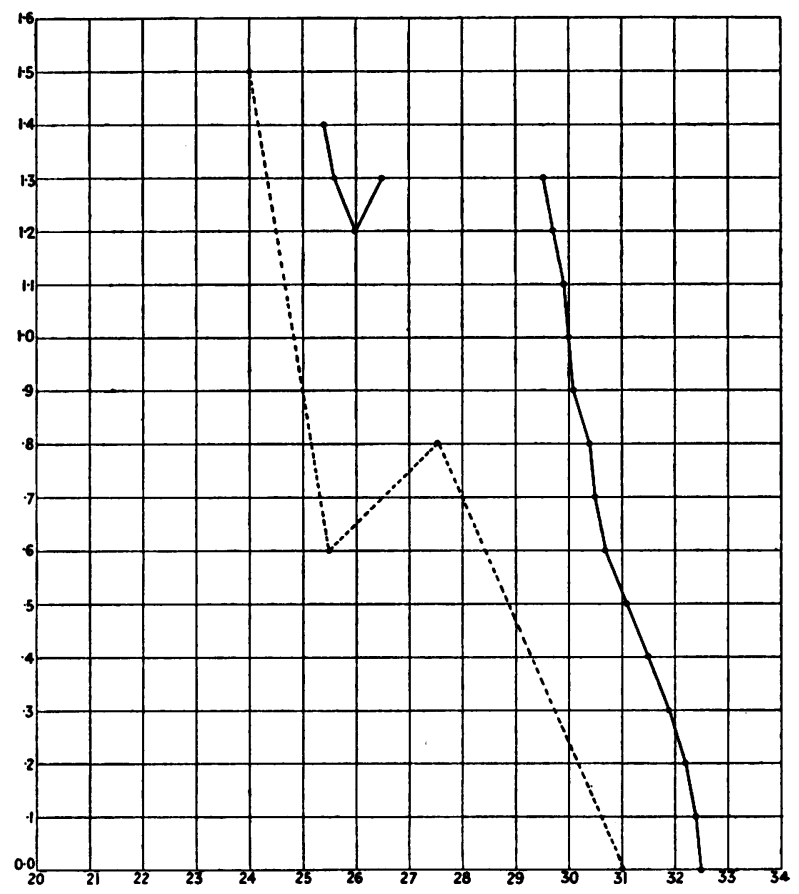

Fig. 5.-Tuberculous meningitis, early stage.

those cases where it is seen), but usually death supervenes and prevents continued observations.

That this is so is seen in fig. 4, which shows the hump, present in fig. 5 , to have completely disappeared. These two graphs are from the same case, fig. 5 being the earlier state and fig. 4 that of a week later.

This persistence of a few lines seems to occur in this situation early in the disease and a similar persistence is found in many cases of general paralysis -as will be noted later. On the whole however the characteristic absorption by cerebrospinal fluid from cases of tuberculous meningitis is that seen in fig. 4. A large number of specimens of fluid from cases of tuberculous meningitis has been examined and the absorption curve is quite constant for any period of the illness and might, I think, be admitted in a doubtful 
case as additional evidence should the question of diagnosis or prognosis be urgent ; it appears to be of about the same evidential value as the chloride content, which tends to become lower the more established the disease. This is seen in figs. 4 and 5 from a case in which the chloride content was at first (fig. 5) found to be $0 \cdot 7$ per cent. and a week later was reduced to $0 \cdot 6$ per cent. (fig. 4).

Turning next to the fluids from cases of general paralysis, a similar constancy of curve was obtained, though, as mentioned before, it is not so

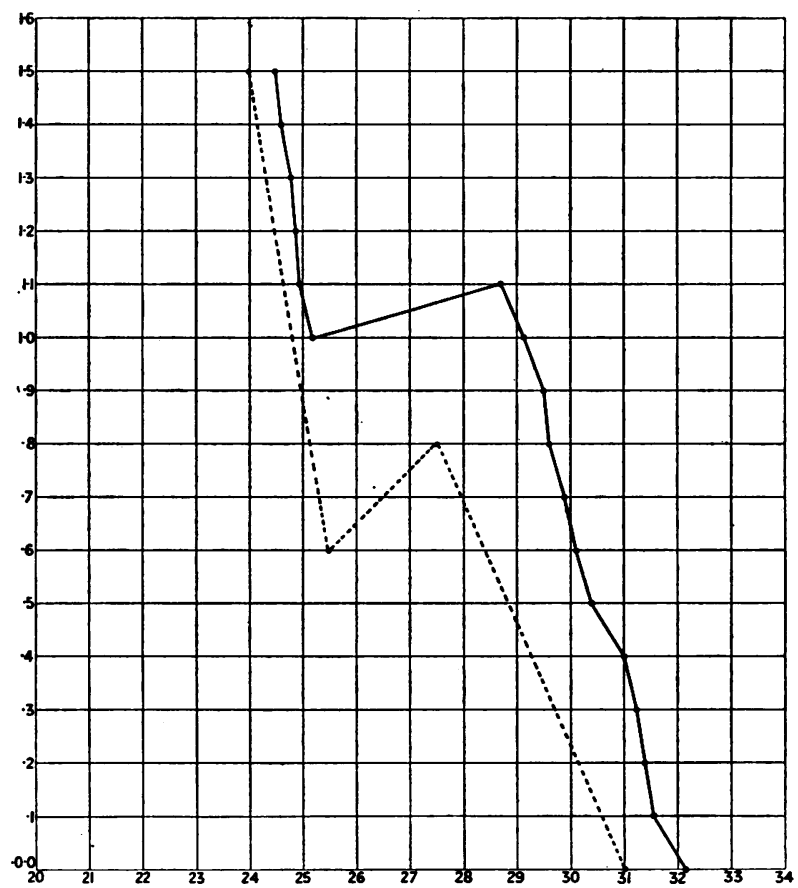

Fig. 6.-General paralysis, early stage.

striking as in cases of tuberculous meningitis. The total absorption is not so complete, beginning at $\lambda 3200$ and becoming less marked as the higher densities are reached, producing a curve less steep than that in the tuberculous cases. The hump too is more frequently seen, though it is shifted towards the top of the graph and in advanced cases disappears altogether, while the total absorption also increases-that is to say, the curve is shifted as a whole towards the region of the long waves.

This last feature of shifting towards the long wave end of the spectrum has been noted by Jacobi and Winkler. ${ }^{5}$

Fig. 6 is the curve of absorption given by cerebrospinal fluid from a case of general paralysis. A large number of such fluids has been examined. A study of these curves brings out one or two points of interest. First, the 


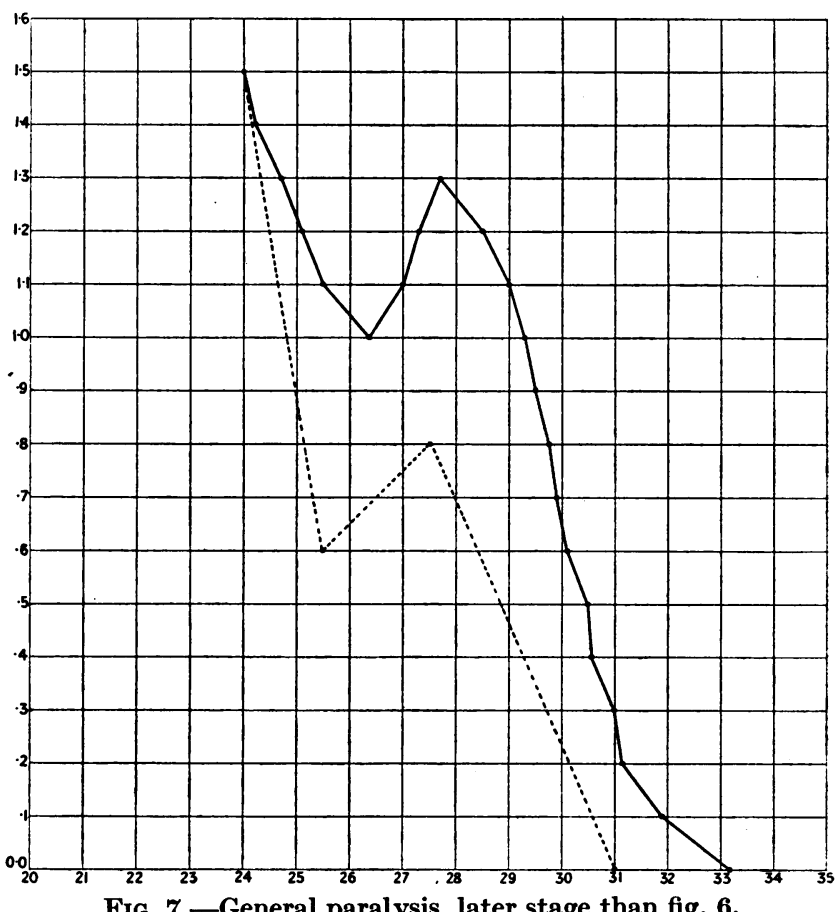

Fig. 7.-General paralysis, later stage than fig. 6.

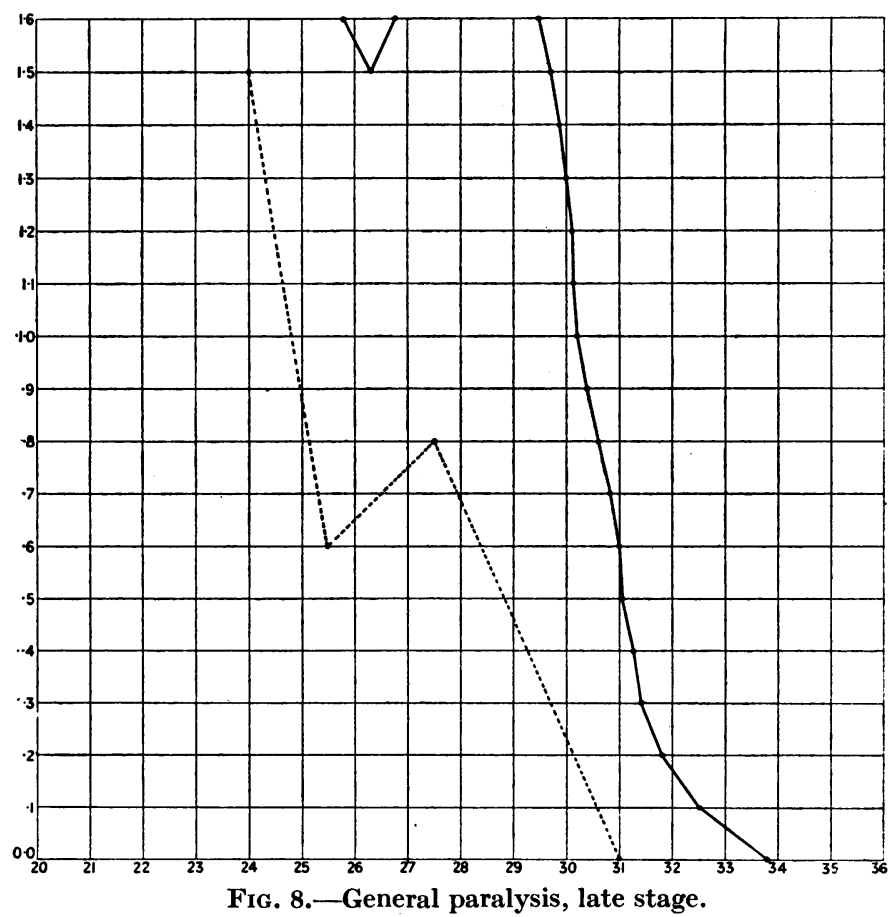


type of curve varies with the stage of the disease, as one would expect; in the early stages being of the same general form as that of normal cerebrospinal fluid except that the first part of the rise is shifted a little to the right and the hump appears a little higher (fig. 6), and then showing more and more departure from this type as the disease progresses.

Fig. 7 is a curve from a case of general paralysis of longer standing, and here the hump is seen to be moving still higher in the graph, though still a

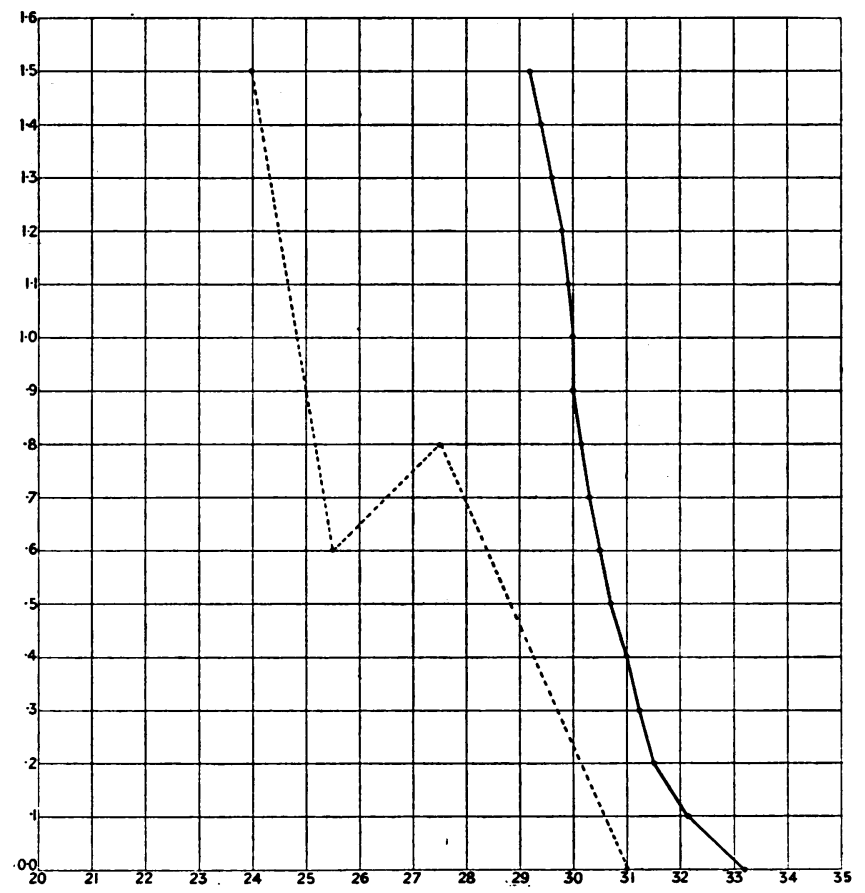

Fig. 9.-General paralysis, late stage.

complete hump; in fig. 8 the hump has moved so high that it is open at the top and has practically disappeared.

In cases of even longer standing, such as the one from which the curve shown in fig. 9 was obtained, the hump has gone completely and the absorption curve is merely a steeply sloping line, i.e. there is total absorption beyond a wavelength of $\lambda 2900$-being almost identical with the type of curve found in tuberculous meningitis.

The treatment of general paralysis by malaria has a marked influence on the curve, as can be seen in fig. 10.

This case was one of long standing and appears to have become more or less stabilized, no progress having occurred for some years. The patient was infected with malaria as long ago as 1929 , and beyond a slight increase of 
globulin in the cerebrospinal fluid this is now almost normal. It will be seen that the absorption has returned towards that found in the early cases, i.e. approximating to the absorption in normal fluid. Jacobi and Winkler ${ }^{5}$ also noted a tendency in the fluid of subjects treated by malaria injections to return towards the normal curve.

The fluid from both groups of cases, tuberculous meningitis and general paralysis, appears to give the same type of absorption curve in the fully developed disease; the differences are probably due to the short time in which meningitis runs its fatal course compared with the long course of

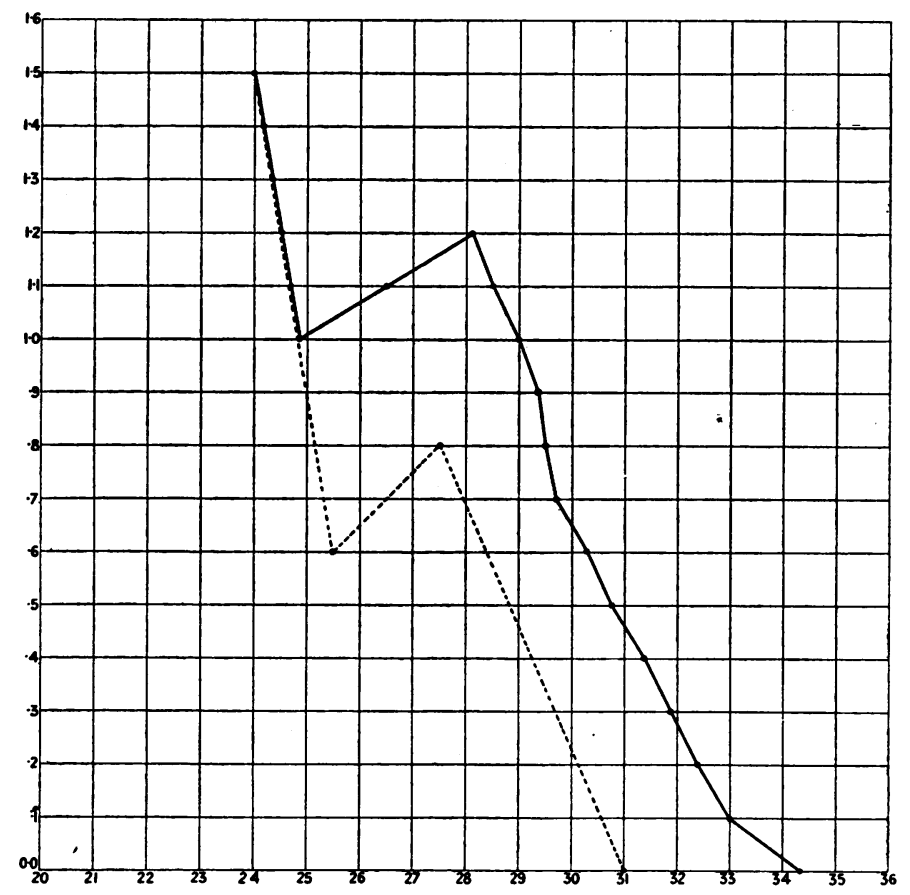

Fig. 10.-General Paralysis after treatment by malaria.

general paralysis and also to the complete absence of any change brought about by treatment in the former, whereas the effect of treatment, and possibly in some cases natural healing processes, tend to alter the appearances of the curve in the latter.

Both these clinical conditions are associated with a pleocytosis, and it seemed desirable to ascertain whether this pleocytosis per se exercised any influence on the absorption curve.

This was done by selecting a fairly early case of general paralysis in which pleocytosis was marked and plotting the curve, and then centrifuging the fluid and again plotting the curve. Fig. 11 shows these two observations on one graph, and it will be readily seen that the cell content of the fluid 
has no influence on the curve, which is in accordance with my previous conclusions. ${ }^{6}$

I had not expected to find that such pleocytosis would produce alteration in absorption for two reasons, firstly because absorption is not influenced by suspended matter unless the latter is in such concentration as to bring about almost complete dispersion of light; and secondly because I had

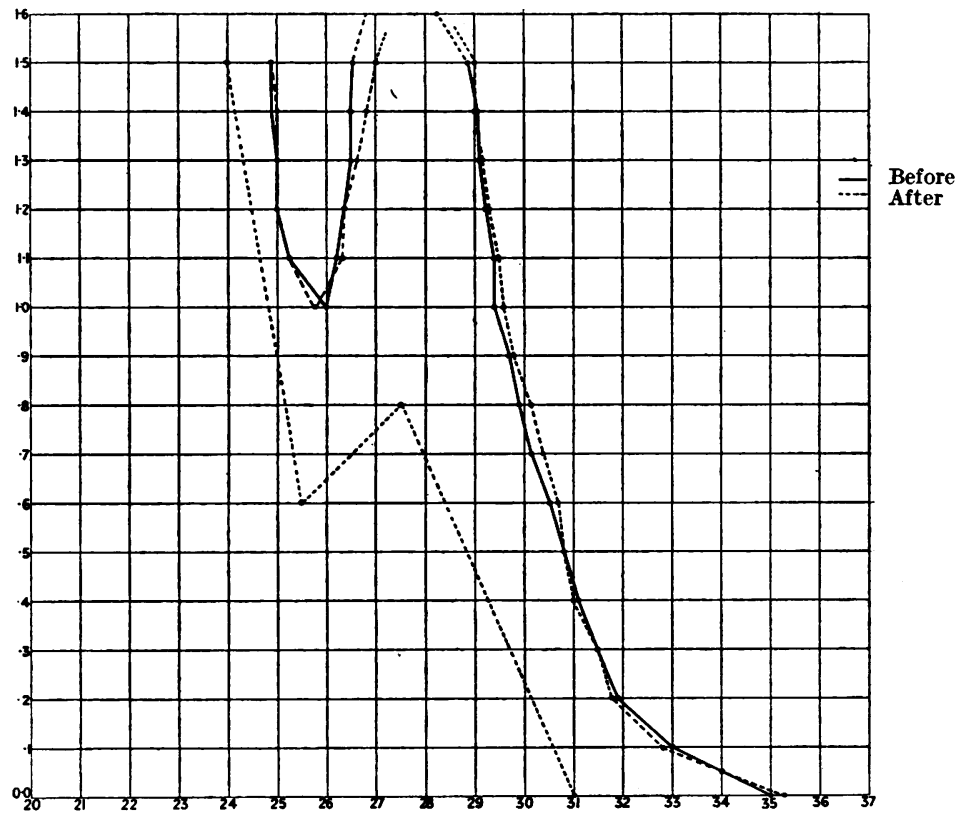

Fig. 11.-Absorption of ultraviolet rays by cerebrospinal fluid before and after centrifuging. Shows that cells have no effect on absorption.

examined a sufficient number of fluids to feel quite sure that there existed no positive correlation between cell content and absorption.

\section{OBSERVATIONS ON DIALYSED GEREBROSPINAL FLUID}

The examination of a large number of absorption spectra has led to the conclusion that, in disease, changes in the absorption curve show themselves in two ways : (1) a general shift of the absorbed areas to the long wave end of the spectrum ; and (2) the disappearance of the characteristic hump seen in normal fluid.

This hump is indicative of selective absorption and in a normal curve means that there is a wide band of absorption between $\lambda 2600$ and $\lambda 3200$, then absorption ceases between $\lambda 2600$ and $\lambda 2400$ when again rays are absorbéd. Were it possible to extend the observations it is likely that similar bands would be found farther along the electromagnetic scale. Repeated observations of over 200 fluids make it clear that the absorption 
between $\lambda 2600$ and $\lambda 3200$ is a biochemical factor of normal fluid and any alteration in its position is pathological.

Since the presence of a large number of cells exerts no influence on the absorption curve, it appeared essential to try and eliminate the other possible factors that might influence it. Cerebrospinal fluid consists essentially of a dilute blood-plasma and contains normally small amounts of salts and proteins, so that separation of these two groups of constituents was the next step.

The separation of salts was attempted first, and here a considerable amount of difficulty was encountered, owing to the fact that only small amounts of fluid are ever available and that the fluid very quickly becomes cloudy. Unless all the apparatus is kept sterile the time required for dialysis to occur (usually 24 to 36 hours) is sufficient to allow contamination by organisms, with the production of turbidity and completely misleading absorption spectra.

The separation of these electrolytes was carried out by dialysis through a collodion membrane at ordinary temperatures and atmospheric pressure so far as the surrounding medium (distilled water) was concerned, the membrane being made in the form of a small bag. It was found that a 5 per cent. collodion in ether and alcohol gave a membrane sufficiently strong to withstand the osmotic pressure and yet tough enough to be handled with care.

The cerebrospinal fluid is put into the dialysing bag with a sterile 5 c.cm. Wright's pipette, the open end of the tube being closed with a tightfitting rubber bung. This bung must have a small tube through it (I used a wide-bored needle) to allow of escape of air when being forced into the tube, otherwise the collodion bag will burst. This air-vent is easily closed by a small rubber tube which is bent on itself and clipped with a screw clamp.

The complete separation of the electrolytes was assumed when all chlorides were gone, and this was ascertained by taking a drop of cerebrospinal fluid in a sterile pipette of fine bore, and dropping it on to a microscope slide whereon was a drop of 20 per cent. silver nitrate solution. If the slide is held above a black card and looked at from above, the light falling on it at an angle, the minutest trace of a precipitate is visible, and $I$ found this very simple technique sufficiently accurate to give constant results.

Fig. 12 is a curve obtained from fluid which was examined both before and after dialysis. The black (righthand) line is the absorption curve before dialysis, while the broken line (the lefthand curve) is that obtained after separation of the electrolytes. The dotted line is the normal curve of absorption.

After removal of the contained salts, by dialysis, the absorption is very much diminished, and, as will be seen from the graph, the hump has gone completely.

From my observations, it appeared that the typical humped curve of normal cerebrospinal fluid was due to the presence of the electrolytes in the 
fluid, and that the removal of these tended to cause a disappearance of this hump - a flattening of the curve towards the short waves. In other words, the selective absorption gives place to a general absorption beginning somewhere about $\lambda 2350$, but $I$ have been unable to measure it accurately as it is just about the lower limit of the apparatus I was using. This result was surprising and quite different from what I had expected. The most striking changes in the absorption curve $I$ had found had been in tuberculous meningitis, as mentioned above, where the curve is moved towards the long wave end of the spectrum, and it is in these fluids that there is a definite and well-recognized

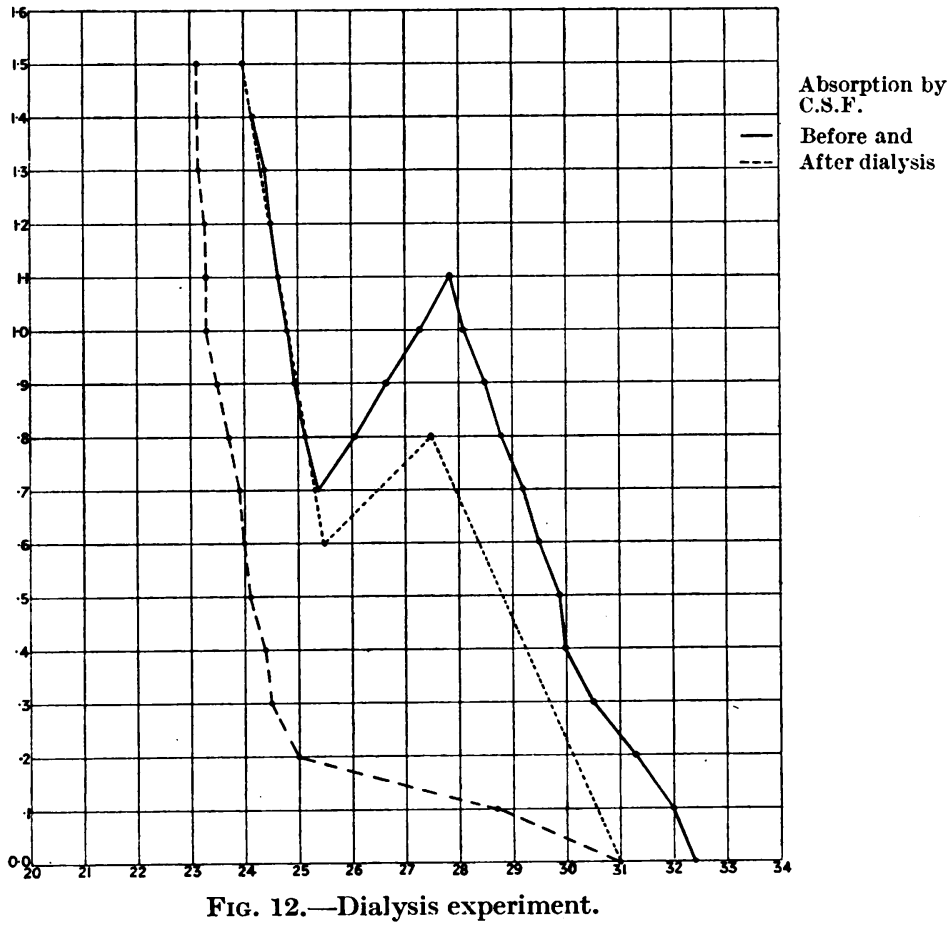

diminution in the chloride content ; hence, if my observations were correct, and I had been at some pains to make them as reliable as possible, this fluid would be expected to produce a curve similar to that in dialysed cerebrospinal fluid, which was obviously not the case. An explanation of this contradictory result might be found in a suggestion that there is some interaction between some of the electrolytes and other constituents of the fluid which may be responsible for the right-ward (long wave) shift in tuberculous cases, or that the influence of some such normal interaction which keeps the absorption curve towards the short wave end of the spectrum is removed and the remaining constituents are able, in the absence of electrolytes, to absorb more of the ultraviolet waves. 


\section{FILTRATION OF GEREBROSPINAL FLUID}

Another series of observations was carried out in an attempt to ascertain the type of curve obtained after the proteins had been removed by precipitation and subsequent filtration.

Here, again, the difficulty of dealing with small quantities of fluid and the necessity for keeping everything sterile became of still greater importance than in dialysis, since the manipulations were more frequent.

I used a small Chamberland filter candle about the diameter of a leadpencil, and enlarged the bore at the open end, into which I placed the tapered end of a small 'thistle' funnel, connected to the candle with a short piece of rubber tube-merely to hold the two together. The candle was passed through a rubber bung which fitted tightly into a filter-flask with a side tube connected to the usual exhaust water-pump. The candle was then dipped into a small wide receiving tube placed inside the flask.

Before use, all this apparatus was sterilized and the small receiving tube, candle, and glass funnel were boiled in distilled water to ensure the absence of any added electrolytes, and distilled water was drawn through the filter for an hour to clear the candle of any salts, as far as possible.

The cerebrospinal fluid was boiled to precipitate the proteins (thus avoiding the addition of other electrolytes in the 'salting out' method). With a long sterile Wright's pipette, cerebrospinal fluid was passed down the bore of the candle (to displace air) and then about $3 \mathrm{c.cm}$. put into the funnel. (At least $3 \mathrm{c.cm}$. are essential, since some is lost in the filter, and one requires $1.5 \mathrm{c} . \mathrm{cm}$. to ensure comfortable filling of the cells before spectroscopy.) Since another 1.5-2 c.cm. are required for the control spectroscopic examination and at least $5 \mathrm{c.cm}$. for the other routine tests, cell count, etc., the use of these very small filter candles is essential, as one cannot safely remove more than 8-10 c.cm. of cerebrospinal fluid as a routine measure.

Fig. 13 shows the type of curve resulting from spectroscopy in such an observation $(a)$ before and $(b)$ after filtration.

A number of such observations was made, and, as will be seen, the general appearance of these graphs (as exemplified in the one above) bears a close resemblance to the curves of meningitic fluids. In a case of meningitis following infection through a fractured base of the skull the curves obtained before and after filtration were so similar that removal of the protein appeared to have had very little if any effect.

The above observations seemed to point to the following conclusions.

1. Normal fluid absorbs the ultraviolet waves in a region between $\lambda 2600$ and $\lambda 3150$, giving the graphed curve a hump in this region.

2. Removal of electrolytes by dialysis prevents this absorption, as shown by the obliteration of the hump, the whole curve becoming horizontal and moved towards the left.

3. Removal of proteins has the opposite effect. It obliterates the hump by increasing the absorption and moves the curve to the right. 
It will be noted that these conclusions are not what would have been expected from the observations obtained in various disease conditions noted previously. For example, in cases of tuberculous meningitis and general paralysis where the fluids have been more highly albuminous than normal, the curve of absorption shifts to the right and the hump is raised until the curve is practically vertical-in other words, a very similar type of curve to - that obtained after removal of the proteins. It would appear then that highly albuminous fluids produce absorption of the same type as do fluids experimentally lacking in protein.

This discrepancy is very real and is difficult to explain, assuming that

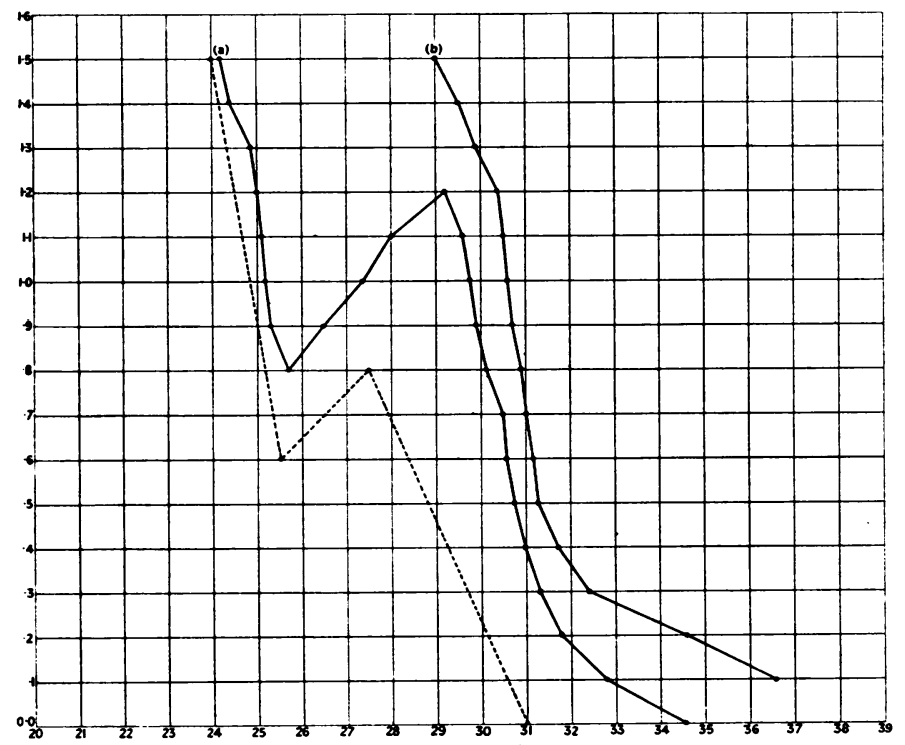

Fig. 13.-Filtration experiment. Absorption of ultraviolet waves $(a)$ before filtration, (b) after filtration.

the technique of filtration is dependable. It may be that the presence of protein molecules holds electrolytes in such a way as to allow of a maximum absorption and that upset in this combination results in alteration in absorptive powers. Even this hypothesis is not completely satisfactory. Since from observations it follows that removal of protein produces a steeper curve, i.e. more complete absorption, it would seem that this must be due to the influence of the remaining electrolytes. Yet in tuberculous meningitis, where this extreme type of curve is found, the reduction of electrolytes is a definite factor, as shown by the diminished amount of chlorides present at any rate in the later stages.

The technique is admittedly open to criticism, and each observation must be made with a freshly cleaned filter, otherwise the most extraordinary results are obtained. The pores of the filter candle become choked with 
albuminous material and may actually add matter to the fluid instead of removing it.

Allowing for this difficulty, however, there remains sufficient concordance to suggest that there is some tendency to obliterate the absorption hump and shift the curve towards the long wave end of the spectrum.

\section{ANALYSIS OF NORMAL GEREBROSPINAL FLUID ABSORPTION GURVE}

The most outstanding feature of normal fluid is the presence of the band of absorption between $\lambda 2600$ and $\lambda \mathbf{3 1 5 0}$, giving rise to the hump in the curve.

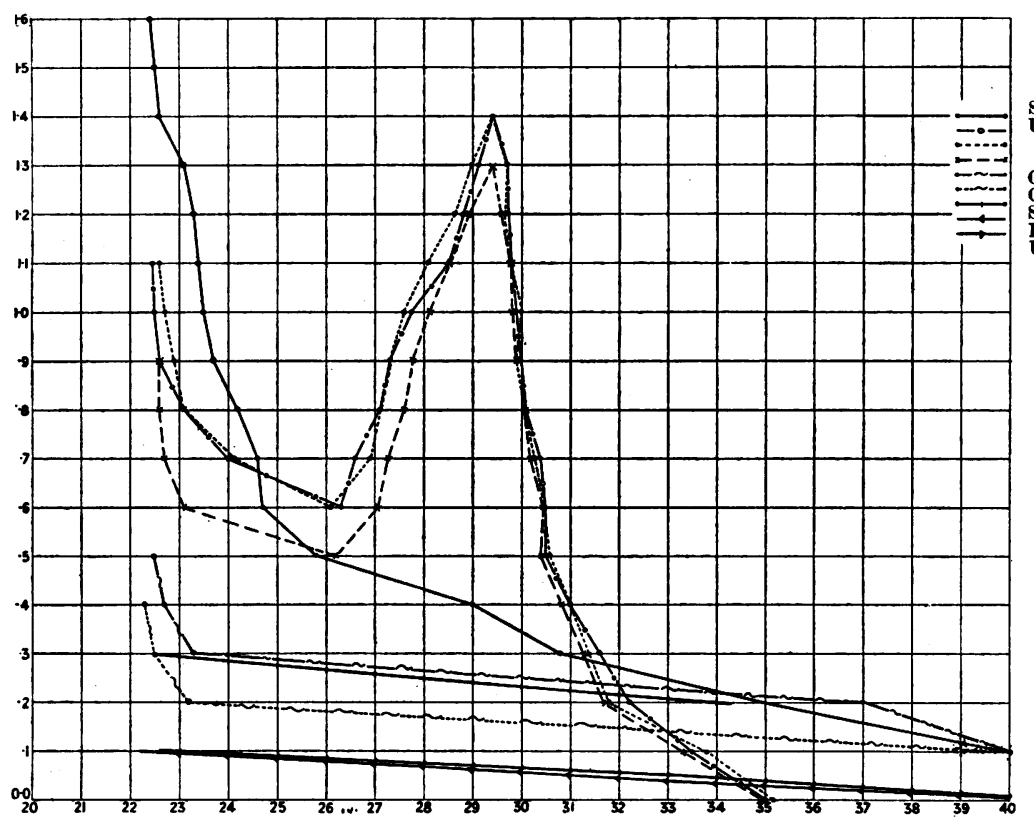

Serum protein 30 mg. $\%$ Uric acid sol. $1 \mathrm{mg} . \%$ No. 1.

FIG. 14.-Absorption of ultraviolet rays by the various constituents of C.S.F. dissolved in distilled water in the concentration found in normal cerebrospinal fluid.

When these experiments were first planned I thought that any alteration in the fluid would almost certainly be due to increase in the protein content and that the proteins would be likely to impart any characteristic feature of absorption. After working for some time, however, it became clear that such a priori views were wrong and an attempt was made to try and ascertain to what the hump was due.

After reviewing various statements about the chemical constitution of cerebrospinal fluid I selected the composition as given by Greenfield and Carmichael 7 as probably the most recent.

I proceeded to examine the spectrogram of each of the constituents, and fig. 14 is a composite graph of these absorption spectra. 
It will be noted that with one exception all the electrolytes give a very similar absorption, which is almost nil, the curve being practically a horizontal line. The proteins again begin to show absorption only in the short wavelength region, similar to the electrolytes just mentioned, but there is a striking absorption between $\lambda 2600$ and $\lambda \mathbf{3 2 0 0}$.

This was given by the last solution submitted to the test, namely, uric acid, a constituent which in some analyses is not even mentioned, and in those analyses which do mention it it is stated to be present in such small amounts as to be negligible. The spectrogram that I obtained was so different

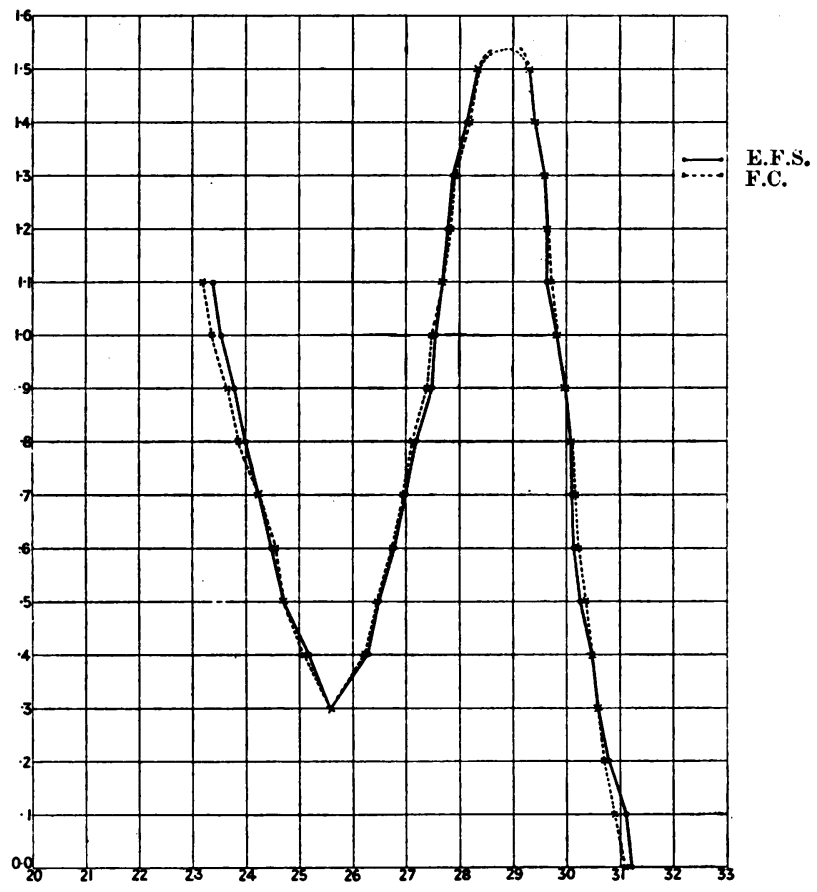

Fig. 15.-Absorption of ultraviolet waves by solution of uric acid (0.01 per cent.). Two observations on same sample of fluid by independent observers.

from what I had expected that I made repeated observations, but always with the same result. Three such observations are recorded on the graph in fig. 14 .

The spectrophotometric analysis of solutions is not a particularly easy technique, and, as will be gathered from the description of the 'spotting' of negatives in the introduction, it depends on careful visual comparison of two slightly dissimilar degrees of photographic blackness. I realized the possibility of personal error in such examination, and in order to test what such error might be, I 'spotted' a spectrogram of a solution of uric acid 0.01 per cent. on the back of the negative, recorded this 'spotting,' and 
rubbed off the spots. I then sent the negative to Dr. Simeon, one of the senior physicists at Messrs. Adam Hilger's, and he kindly allowed one of his trained staff to 'spot' the negative again. The negative was unmarked in any way. Fig. 15 represents the graph constructed from the two independent examinations of this negative.

It appears clear from this that the difference in personal variation of two individuals 'spotting' the same negative is very slight. I think therefore one can claim that this technique, once acquired, is probably less liable to

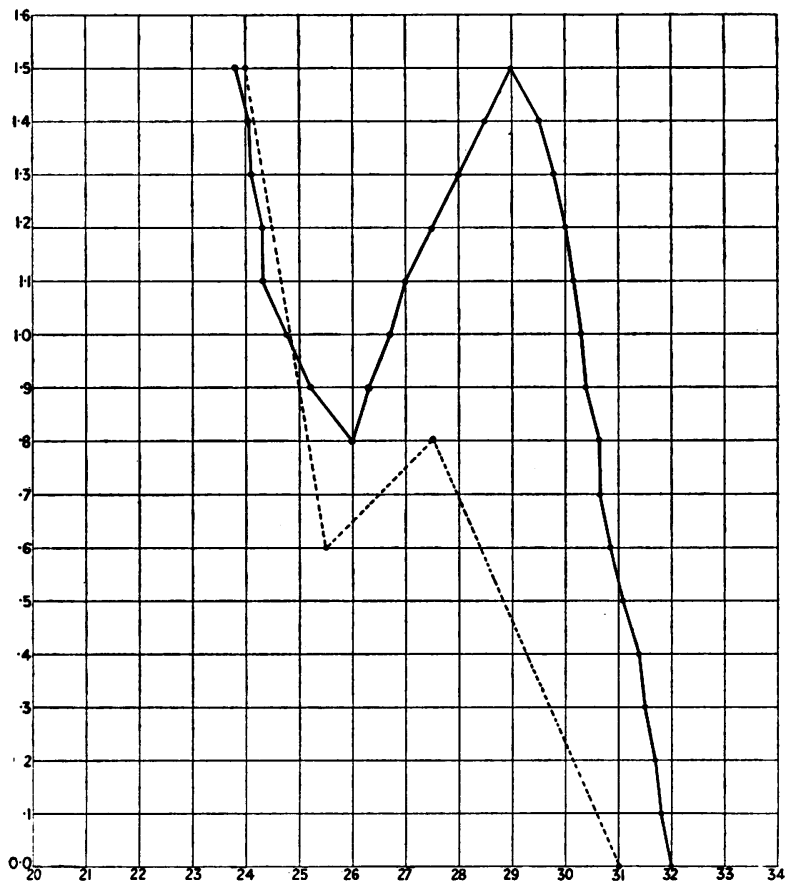

Fig. 16.-Absorption of ultraviolet waves by serum of normal blood. Absorption due to uric acid (after Campbell Smith). Similarity of absorption by C.S.F. suggests the same cause.

personal error than the estimation of, say, hæmoglobin in a routine blood count.

After a number of observations had been made from both normal and pathological fluids, it appeared justifiable to assume that the absorption of ultraviolet waves by cerebrospinal fluid in the region of wavelengths between $\lambda 2600$ and $\lambda 3200$ might be directly attributable to the minute traces of uric acid occurring in the fluid. If this absorption band, so noticeable in normal fluid, is due to uric acid (as seems possible), an increase in the amount present would be likely to increase the absorptive power of the fluid. Such an increase would tend to obliterate the hump in the curve and render it steeper by 
shifting the curve towards the right end of the spectrum. In other words, there would be a tendency for an increased amount of uric acid to give rise to the meningitic type of curve.

In this connexion it is of interest to note Bernhard's ${ }^{8}$ findings. In a series of 51 cases he has found that "The average uric acid content, excluding cases of meningitis, is rather higher in children than in adults. In polioencephalitis and encephalitis lethargica rather high normal figures were found, e.g. 0.8, $0.88,1.2$ and $2 \mathrm{mgm}$., while in meningitis the readings were occasionally much higher still, up to 3.5 and $4.8 \mathrm{mgm}$.'

If one can accept the uric acid content as the cause of the shifting of the curve to the right, then the discrepancy between the observations of dialysis and filtration, noted previously, is largely explicable. Dialysis removes the salts, including uric acid, and a more or less horizontal curve is obtained (fig. 14) with a shift to the left, any remaining absorption being due to the protein. Filtration, on the other hand, removes the protein, leaving the salts, and the uric acid may now be able to exert more influence than when it is present in conjunction with protein in normal amount. In meningitis the absorptive effect of uric acid appears to outweigh that of the protein, although both constituents may be increased.

This type of curve, which on the evidence above might be assumed to be due to increased uric acid in the cerebrospinal fluid, is always associated clinically with coma, and it may be that the coma is a direct toxic action on the neurons of this high concentration of uric acid. It is of interest to note that a similar type of curve is found in cases of uræmia. Examination of a number of fluids from fatal cases of coma of various types-uræmia, cerebral hæmorrhage, lateral sinus thrombosis, etc.-gave absorption curves so similar to the meningitic curve that there arises the possibility that they may be all due to one factor and that uric acid may be the factor involved.

Campbell Smith, ${ }^{9}$ working with blood serum, in 1928 found a curve of absorption for uric acid practically identical with the ones I have depicted above. In his work he filtered serum through a special membrane by a process of rapid dialysis, ${ }^{10}$ and the resulting filtrate gave the absorption curve shown in fig. 16. By such a rapid method it is likely that some of the smaller protein molecules may have got through, as well as all the electrolytes, so that he was really producing a fluid practically identical with cerebrospinal fluid, which is, after all, a filtrate of blood serum through a similar membrane.

\section{MISCELLANEOUS GASES}

In January, 1935, there was admitted to one of my wards a young woman of about 25 years of age, deeply comatose, and of whom little could be learned at the time. She had been found unconscious at her work.

A lumbar puncture was done at once, and on examining the fluid spectro- 
graphically I obtained a curve rather like the early meningitic or syphilitic curve, but in its position on the graph also resembling the 'coma' type. I was at first convinced of the uræmic nature of the coma, but clinical observation did not bear this out, and as she began to recover after about two days the diagnosis was left open.

It was learned later that she had tried to procure abortion (she was about three months pregnant) by taking some 'stuff' given to her by her partner. She had no knowledge of what this 'stuff' was, nor could we get any evidence from her parents.

The similarity of her condition and that of patients recovering from the effects of nembutal anæsthesia suggested that she might have been given one of this group of drugs. Accordingly I tried to arrange with a colleague, who has a licence, to inject nembutal into a rabbit and then draw off the cerebrospinal fluid and allow me to examine it with the spectrograph. Owing, however, to technical difficulties the observation was abandoned and I had to content myself with a substitute for it. I obtained several samples of normal cerebrospinal fluid and added to them varying percentages of both veronal and nembutal and then examined the spectrogram of each solution. A composite graph of this observation gave curves almost identical with that obtained from the comatose girl. It seemed likely therefore that she had been poisoned by one of the barbituric group, and this was afterwards confirmed by my colleague Dr. Webster, Police Surgeon for the City of Sheffield, who from other evidence had obtained proof of barbituric acid poisoning.

This observation seemed interesting in suggesting that with adequate safeguards and an assured technique the method might have a medicolegal value in the identification of drugs excreted by body fluids.

In hydrocephalic cerebrospinal fluid, in which the constituents are present in more minute amounts than normal (the fluid consisting practically of water), the type of curve is interesting, being similar to that obtained in a normal fluid after dialysis when all the protein is removed. There must be a trace of protein in the hydrocephalic fluid since filtration after boiling does make a slight difference, and that in the usual shift of the curve to the right.

Although only three or four observations have been made on hydrocephalic fluids as opportunity has offered, this fluid is so different from normal cerebrospinal fluid that $I$ have introduced the observation as it appears to me to support the experimental results in dialysis and filtration mentioned above.

Several examinations have been made of fluid from cases of schizophrenia, but I have as yet failed to notice any definite characteristic in these cases. This is more or less in conformity with the conclusions of Jacobi and Winkler. ${ }^{5}$ Unfortunately these two observers produce curves which, as they themselves admit, cannot be compared with those of other workers, 
since instead of using the extinction coefficient as ordinate they employ the logarithm of the varying thickness of the fluid through which the light is passed.

In a more recent paper they ${ }^{11}$ express the opinion that in early cases of schizophrenia the absorptive power is not very different from that of normal people, as I have found in the few cases I have examined, but that later in the progress of such cases the fluid does show changes in the direction of marked absorption increase. They conclude their paper by stating : 'Although our findings are not sufficiently secure to be used as a method of differential diagnosis, nevertheless we think that they have a special interest in connexion with the view gradually gaining ground that the schizophrenic process is associated with definite somatic changes.'

\section{GONGLUSIONS}

From the observations made in these experiments it appears that certain conclusions can be drawn; and though with better apparatus some of the details would probably be varied, the general tendency will, I think, be found to hold.

1. Normal cerebrospinal fluid has a definite absorption spectrum with a band of absorption between $\lambda 2600$ and $\lambda 3200$, which gives a humped curve on a graph.

2. In cases of infection of the meninges, whether acute or chronic, the normal curve is shifted towards the long wave end of the spectrum.

3. There appear grounds for believing that the hump in the normal curve may be due to uric acid and that the shift to the right in inflammatory conditions is possibly due to an increase in this constituent.

4. Certain observations seem to suggest that in some cases coma may be due to the direct action of uric acid on neurons.

5. Removal of electrolytes diminishes absorption and shifts the curve to the left, giving a horizontal-seeking curve.

6. Removal of protein has the opposite effect, giving increased absorption and a shift of the curve to the right.

7. There is some slight evidence that the method of ultraviolet spectroscopy might be of use in identifying substances excreted in body fluids.

I would particularly like to record my thanks to Dr. Simeon of Messrs. Adam Hilger's for much kindly help in the early stages of my experiments.

\section{REFERENCES}

1 Skinner, E. F., Jour. Neurol. and Psychopathol., 1930, 11, 144.

2 Skinner, E. F., Jour. Neurol. and Psychopathol., 1929, 10, 97.

3 Bunsen and Roscoe, Pogg. Ann. d. Physik u. Chemie, 177, 238.

4 Hurter, F. and Driffield, V. C., Jour. Soc. Chem. Industry, 1890, 455.

5 Jacobi and Winkure, Deut. Zeits. f. Nervenheilk., 1929, $111,16$. 
- Skinner, E. F., Jour. Neurol. and Psychopathol., 1930, 11, 149.

7 Greenfield, J. G., and Carmichael, E. A., The Cerebrospinal Fluid in Clinical Diagnosis, 1925, 44.

3 Bernhard, quoted by Greenfield and Carmichael (supra).

- Campbell Smith, Biochem. Jour., 1028, 22, 1499.

10 id., Jour. of Physiol., 1928. 65.

11 Jacobi and Winkler, Archiv f. Psychiat., 1930, 91, 171. 